\title{
Editorial
}

\section{Advances in Finite Element Method 2014}

\author{
Song Cen, ${ }^{1}$ Chenfeng Li, ${ }^{2}$ Sellakkutti Rajendran, ${ }^{3}$ and Zhiqiang $\mathrm{Hu}^{4}$ \\ ${ }^{1}$ AML, Department of Engineering Mechanics, School of Aerospace Engineering, Tsinghua University, Beijing 100084, China \\ ${ }^{2}$ Zienkiewicz Centre for Computational Engineering \& Energy Safety Research Institute, College of Engineering, Swansea University, \\ Swansea SA2 8PP, UK \\ ${ }^{3}$ School of Mechanical and Aerospace Engineering, Nanyang Technological University, Nanyang Avenue, Singapore 639798 \\ ${ }^{4}$ State Key Laboratory of Ocean Engineering, Shanghai Jiao Tong University, Shanghai 200240, China
}

Correspondence should be addressed to Song Cen; censong@tsinghua.edu.cn

Received 11 March 2015; Accepted 11 March 2015

Copyright (c) 2015 Song Cen et al. This is an open access article distributed under the Creative Commons Attribution License, which permits unrestricted use, distribution, and reproduction in any medium, provided the original work is properly cited.

To date, the finite element method (FEM) is still a prominent technique for most numerical analyses and simulations in science and engineering. Numerous research activities related to the FEM are still actively pursued worldwide. In order to solve various complicated mechanics and mathematical problems more efficiently, many researchers are devoting themselves to developing new principles, techniques, algorithms, models, and schemes to improve precision, efficiency, robustness, and applicability of the FEM. It is significant and necessary to disseminate these latest ideas, developments, and applications to readers all over the world.

After successful organization and publication works for the first issue in 2013, the special issue Advances in Finite Element has been upgraded to be one of the annual issues in the journal. This is a good news for many FEM developers and users because they will continuously have a platform to introduce their latest achievements. The second issue, this issue, was opened during last May to September and attracted more than seventy submissions. In order to improve the quality of the papers published in MPE, the publisher has decided to adopt a much more rigorous review policy. Therefore, the whole review process for these submissions could only be finished by the beginning of March 2015, and forty-two manuscripts finally passed the peer review, including one review article and 41 research articles which have provided a fairly complete coverage of the current research in the development and application of FEM and other related numerical methods. The topics covered by this special issue include the following:

(i) new types of FEM and related numerical techniques, including an extensive review on the development of Mindlin-Reissner plate elements (work of S. Cen and Y. Shang), a hierarchical laminated shell element (work of J. S. Ahn et al.), several improved plane membrane element models (works of G. Shi et al., P.-L. Zhou and S. Cen, X.-M. Chen et al., or X.R. Fu et al.), new beam and bar elements (works of Q. Cao et al. or L. Tang et al.), a technique for analysis of stress field gradient using low-order $\mathrm{C}^{0}$ elements (work of J. Xing and G. Zheng), a hybrid finite element-Fourier spectral method (work of W.$\mathrm{Y}$. Li et al.), and a multibody finite element method (work of C. Su et al.);

(ii) applications of FEM in nonlinear, coupled, dynamic, and fracture problems, including some new methods and techniques for nonlinear problems (works of T. Li et al., X. Deng and M. Liu), coupled problems (works of X. Song and Y. Wang, S. Wang et al., H. Tong et al., Y. Wang et al., or W. Wu et al.), dynamic problems (works of B. Yan et al., L. Zhang et al., or X.-H Zhu et al.), and fracture problems (works of P. Cao et al., D. Liu et al., L. Zhao et al., or F. Liu et al.);

(iii) structural optimization problem (works of $\mathrm{H}$. Ye et al. or C. Su et al.);

(iv) mesh generation (work of S. Sun et al.);

(v) high performance computing (works of X. Fan et al. or J. Zhang);

(vi) new software systems based on FEM, including an inverse analysis system for temperature and stress of concrete dam (work of L. Zhang et al.), an intelligent autofeedback, and safety early-warning system for 
underground cavern engineering (work of L. Xu et al.);

(vii) engineering and Scientific applications, including analyses of compound lining in complex underground surge-shaft structure (work of J. Chen et al.), rock and concrete (works of Y. Peng et al., Y. Peng et al., or J. Zhang et al.), 3D pressure vessel (work of X. Du et al.), flexible risers (work of J.-Y. Li et al.), water flow pressure on bridge piers (work of Y. Wang et al.), thermal characters for natural fiber bundle with numbered lumens (work of G.-Y. Zheng), fatigue characters for wheel considering contact effect (work of Q.-Y. Xiong et al.), and dynamic characters for ultrasonic transducer (work of C. Ge).

We hope that the great diversity of the presented topics covering the state-of-the-art FEM research will give this special issue a much more lasting value and make it appealing to a broad audience of researchers, practitioners, and students who are interested in FEM. We hope that each reader can find in this special issue something useful or inspiring.

\title{
Acknowledgments
}

We would like to thank all the authors of this special issue for their excellent contributions. And we would also like to thank the support from the National Natural Science Foundation of China (Project no. 11272181), the Specialized Research Fund for the Doctoral Program of Higher Education of China (Project no. 20120002110080), and the Tsinghua University Initiative Scientific Research Program (Project no. 2014z09099).

\author{
Song Cen \\ Chenfeng $\mathrm{Li}$ \\ Sellakkutti Rajendran \\ Zhiqiang $\mathrm{Hu}$
}






Advances in

Operations Research

mansans

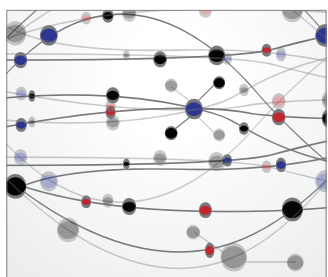

The Scientific World Journal
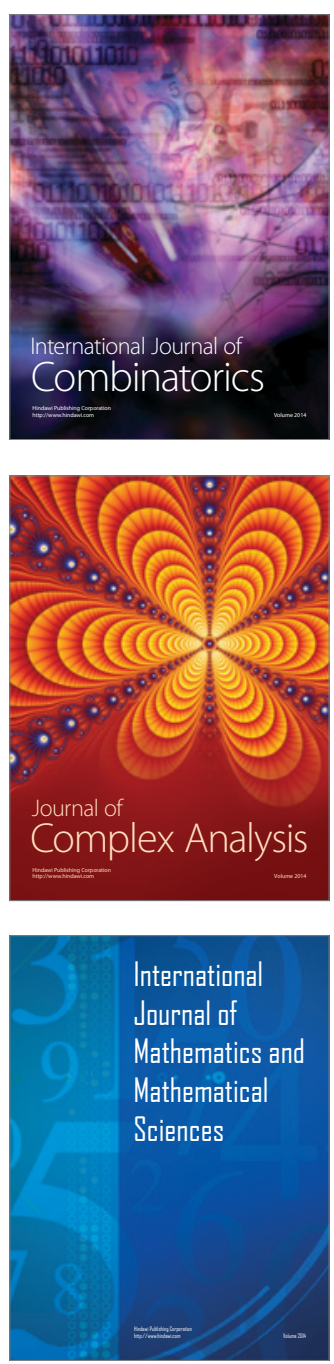
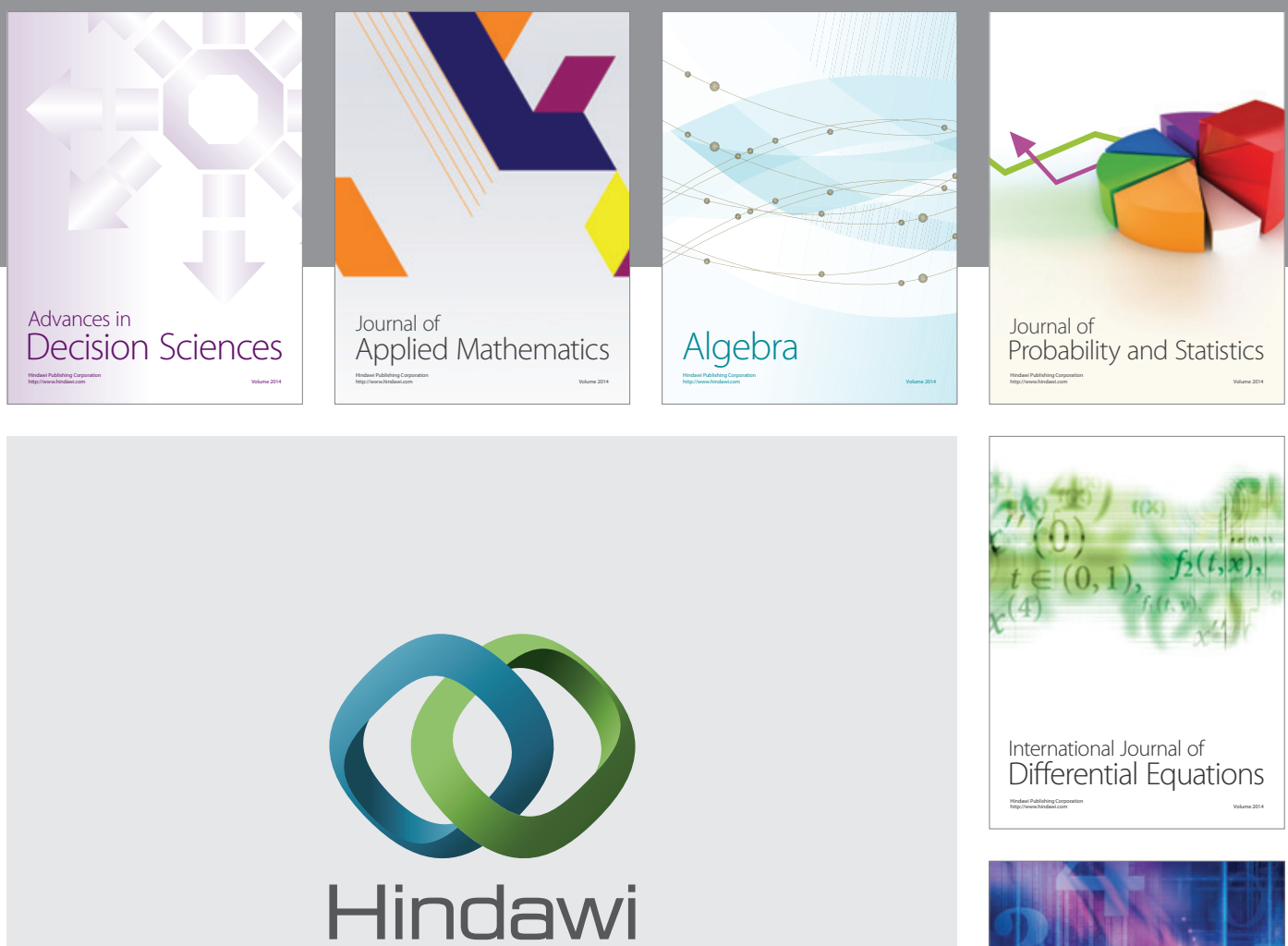

Submit your manuscripts at http://www.hindawi.com
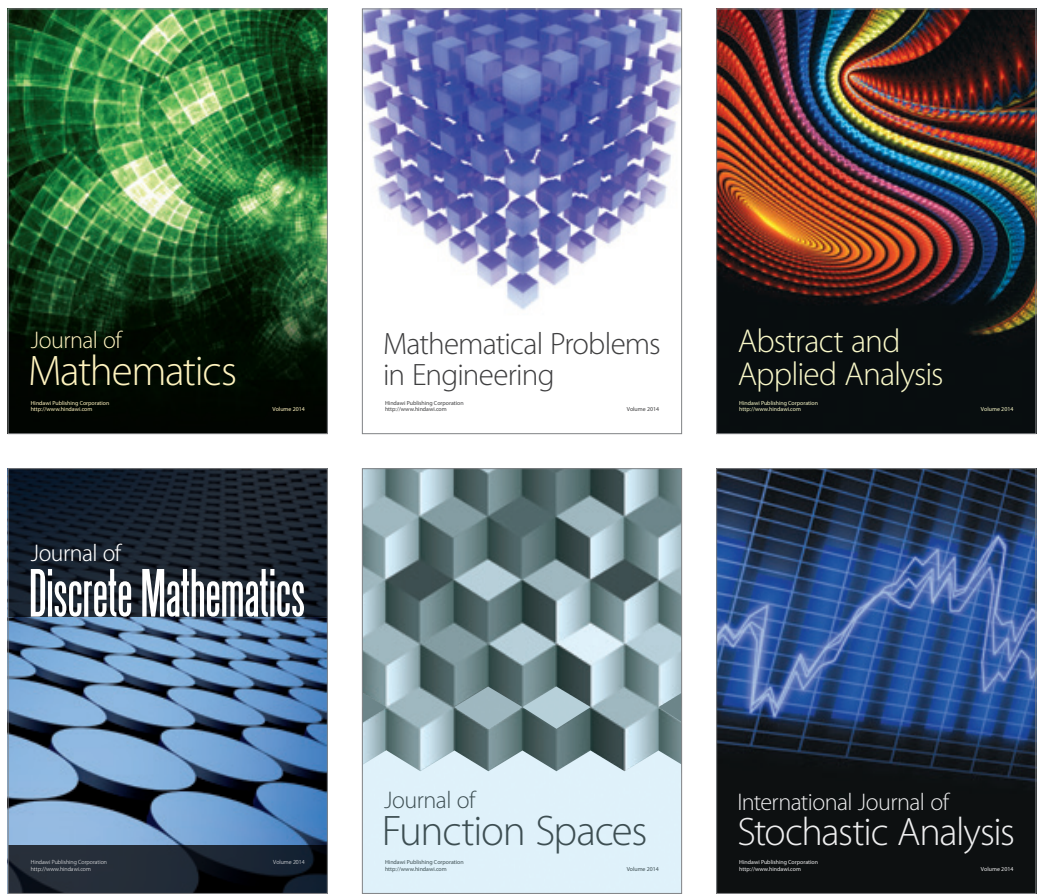

Journal of

Function Spaces

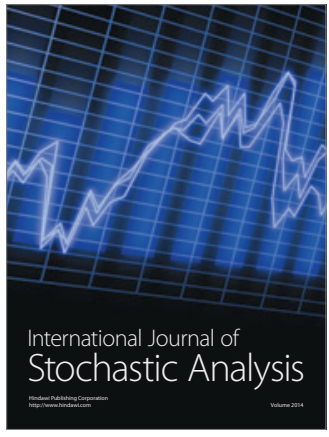

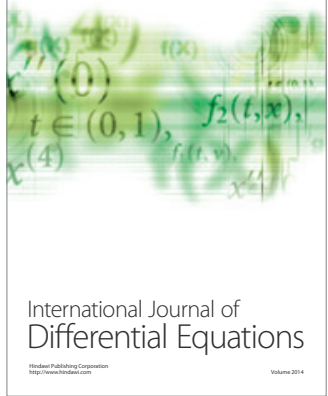
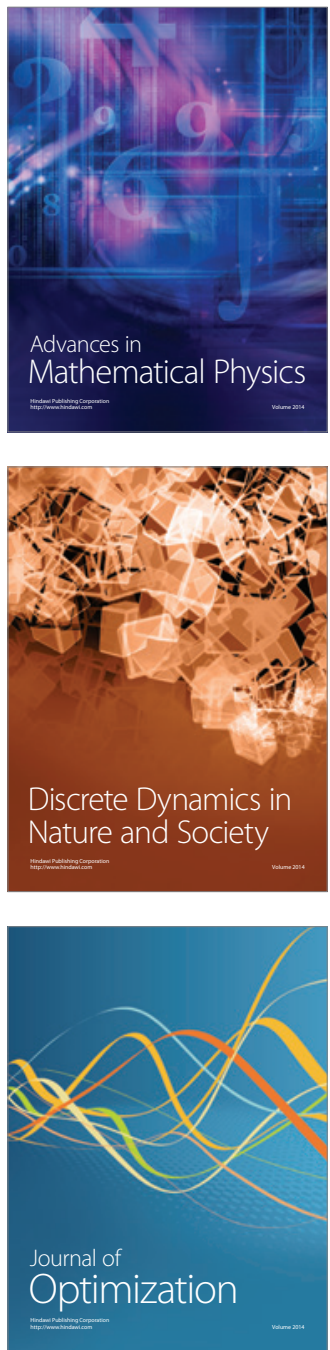